\title{
OBSERVATION OF STRONG-STRONG AND OTHER BEAM-BEAM EFFECTS IN RHIC*
}

\author{
W. Fischer ${ }^{\dagger}$, M. Blaskiewicz, J.M. Brennan, P. Cameron, R. Connolly, C. Montag, S. Peggs, F. Pilat, \\ V. Ptitsyn, S. Tepikian, D. Trbojevic, J. van Zeijts, BNL, Upton, NY 11973, USA
}

\section{Abstract}

RHIC is currently the only hadron collider in which strong-strong beam-beam effects can be seen. For the first time, coherent beam-beam modes were observed in a bunched beam hadron collider. Other beam-beam effects in RHIC were observed in operation and in dedicated experiments with gold ions, deuterons and protons. Observations include measurements of beam-beam induced tune shifts, lifetime and emittance growth measurements with and without beam-beam interaction, and background rates as a function of tunes. During ramps unequal radio frequencies in the two rings cause the crossing points to move longitudinally. Thus bunches experience beam-beam interactions only in intervals and the tunes are modulated. In this article we summarize the most important beam-beam observations made so far.

\section{INTRODUCTION}

The beam-beam interaction is a major consideration in the operation of RHIC. It can lead to emittance growth and particle loss, and is a source for experimental background. Machine parameters, close to the maximum parameters achieved so far, are presented in Tab. 1. RHIC consists of two superconducting rings, Blue and Yellow, and has produced gold-gold, proton-proton and deuterongold collisions [1]. With RHIC's interaction region design (Fig. 1) and with 4 experiments beams experience 4 headon, and 2 long-range collisions per turn. The long-range interactions are with at least $7 \mathrm{rms}$ beams sizes separation. With 120 or less bunches per ring (the current limit), sets of 3 bunches in one ring and 3 bunches in the other ring are coupled through the beam-beam interaction.

Even small tune shifts due to the beam-beam interaction can be observed directly with a high precision tune measurement system [2]. In Fig. 2 a tune shift measurement is shown that was taken 3 hours into a gold-gold store.

Two beam splitting DX dipoles are the magnets closest to the interaction point (IP). They are each $10 \mathrm{~m}$ away from the IP (Fig. 1). Beams collide nominally without a crossing angle. With rf manipulations, the crossing point can be moved longitudinally. If the bunch spacing is large enough (with 60 or less bunches per ring), it is possible to separate the beams longitudinally and switch off all 6 beam-beam interactions. If the crossing point is moved within the $\mathrm{DX}$ magnets, an observed tune shift is a sign of crossing angles (Figs. 1 and 2). The sum of all residual crossing angles is typically about $0.5 \mathrm{mrad}$.

\footnotetext{
* Work supported by US DOE, contract No DE-AC02-98CH10886.

$\dagger$ Wolfram.Fischer@bnl.gov
}

Table 1: Machine parameters relevant to beam-beam interactions, for $\mathrm{Au}-\mathrm{Au}$ and $\mathrm{p}-\mathrm{p}$ collisions.

\begin{tabular}{lccc}
\hline \hline parameter & unit & Au-Au & p-p \\
\hline relativistic $\gamma$, injection & $\ldots$ & 10.5 & 25.9 \\
relativistic $\gamma$, store & $\ldots$ & 107.4 & 106.6 \\
no of bunches $n_{b}$ & $\ldots$ & 55 & 55 \\
ions per bunch $N_{b}$ & $10^{9}$ & 1 & 100 \\
emittance $\epsilon_{N} x, y 95 \%$ & $\mu \mathrm{m}$ & 10 & 20 \\
chromaticities $\left(\xi_{x}, \xi_{y}\right)$ & $\ldots$ & \multicolumn{2}{c}{$(+2,+2)$} \\
harmonic no. $h$, store & $\ldots$ & $7 \times 360$ & 360 \\
synchrotron tune $Q_{s}$ & $10^{-3}$ & 3.0 & 0.5 \\
rms bunch length $\sigma_{z}$ & $\mathrm{~m}$ & 0.3 & 0.7 \\
rms momentum spread $\sigma_{p} / p$ & $10^{-3}$ & 0.15 & 0.3 \\
envelope function at IP $\beta^{*}$ & $\mathrm{~m}$ & \multicolumn{2}{c}{$1-10$} \\
beam-beam $\xi / \mathrm{IP}$ & $\ldots$ & 0.0023 & 0.0037 \\
crossing angle $\theta$ & $\mathrm{mrad}$ & \multicolumn{2}{c}{0.0} \\
head-on collisions & $\ldots$ & \multicolumn{2}{c}{4} \\
parasitic collisions & $\ldots$ & \multicolumn{2}{c}{2} \\
\hline \hline
\end{tabular}

Beam-beam phenomena observed in other hadron colliders [3] can also be seen in RHIC. In addition, with bunches of equal intensity the beams are subject to strong-strong effects. To accommodate acceleration of different species, the two RHIC rings have independent rf systems. With different rf frequencies the beam-beam interaction is modulated and can have a visible impact on the beam lifetime.

The beam-beam tune shift depends on the bunch intensity and emittance. Since the bunch intensities can be measured with good precision, the beam-beam tune shift measurement also provides an emittance estimate.

\section{LIFETIME AND EMITTANCE GROWTH}

The beam-beam interaction is most pronounced for proton-proton collisions with larger $\beta^{*}$ values (Tab. 1). In lattices with small $\beta^{*}$ uncorrected nonlinear field errors in the triplets have a significant impact on the beam lifetime. Fig. 3 shows the distributions of the bunched beam lifetimes for polarized proton collisions during the 2001 run, with $\beta^{*}=3 \mathrm{~m}$ at all IPs. The distributions are relatively

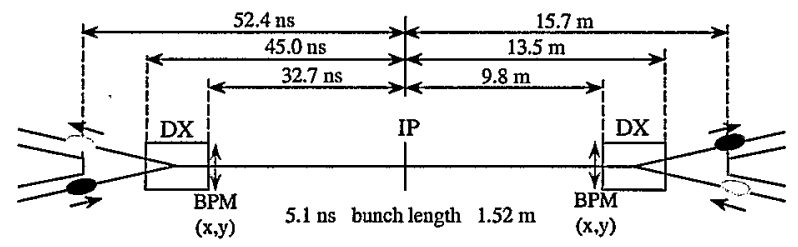

Figure 1: RHIC interaction region. Beams share a common beam pipe between the beam splitting DX dipoles. The bunch spacing shown corresponds to a fill pattern of 120 symmetrically distributed bunches. 


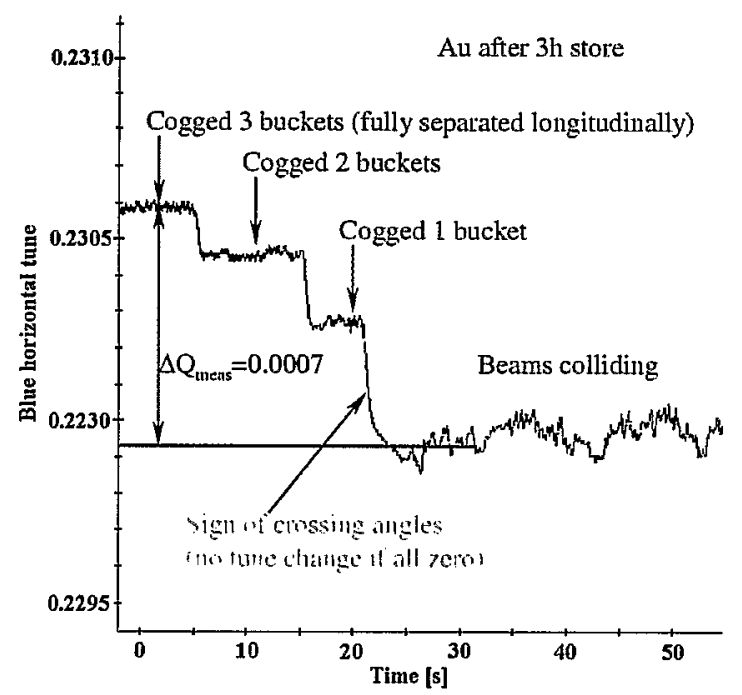

Figure 2: Tune as a function of longitudinal beam crossing position. Cogging by one acceleration bucket moves the crossing point by $5 \mathrm{~m}$.

wide with a mean of about 15 hours. The average bunch intensity for all stores in the plot is $N_{b}=0.4 \cdot 10^{11}$. Gold beam lifetimes are generally lower due to intra-beam scattering. A deuteron beam without collisions, in a lattice with $\beta^{*}=2-10 \mathrm{~m}$, provides a good comparison to see the impact of the beam-beam interaction. Its lifetime is only marginally influenced by intra-beam scattering or nonlinear triplet errors. 55 bunches with $N_{b}=0.5 \cdot 10^{11}$, stored for an hour, showed a lifetime of $830 \mathrm{~h}$.

From the change in the beam intensity and the observed luminosity an estimate for the emittance growth can be obtained, assuming the same emittance in both beams. For proton-proton collisions we find $\Delta \epsilon / \epsilon=4 \%$ in the first store hour (with an rms value of 5\%). For comparison, no emittance growth was observed with the ionization profile monitor in the deuteron beam measurement without beambeam interaction.

\section{WORKING POINT AND BACKGROUND}

Both transverse RHIC fractional tunes $\left(Q_{x}, Q_{y}\right)$ are kept between 0.2 and 0.25 , and during stores close to the coupling resonance $Q_{x}=Q_{y}$. In this area the lowest order

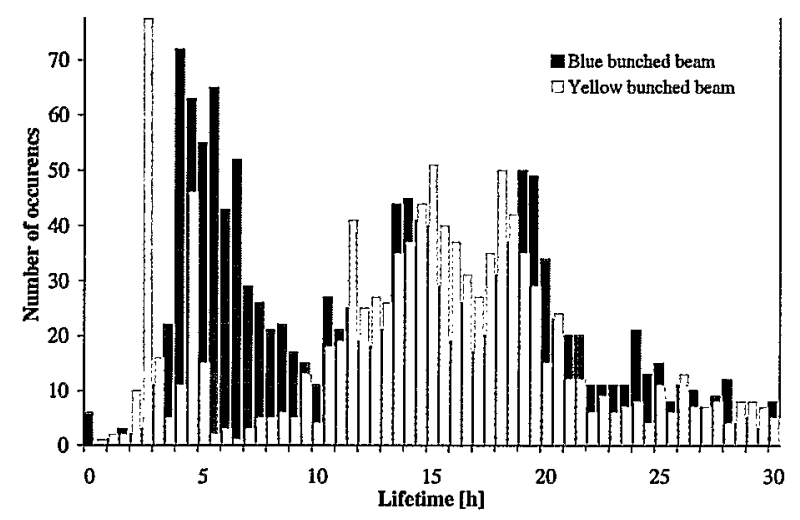

Figure 3: Blue and Yellow bunched beam lifetimes with proton-proton collision during one month of operation.

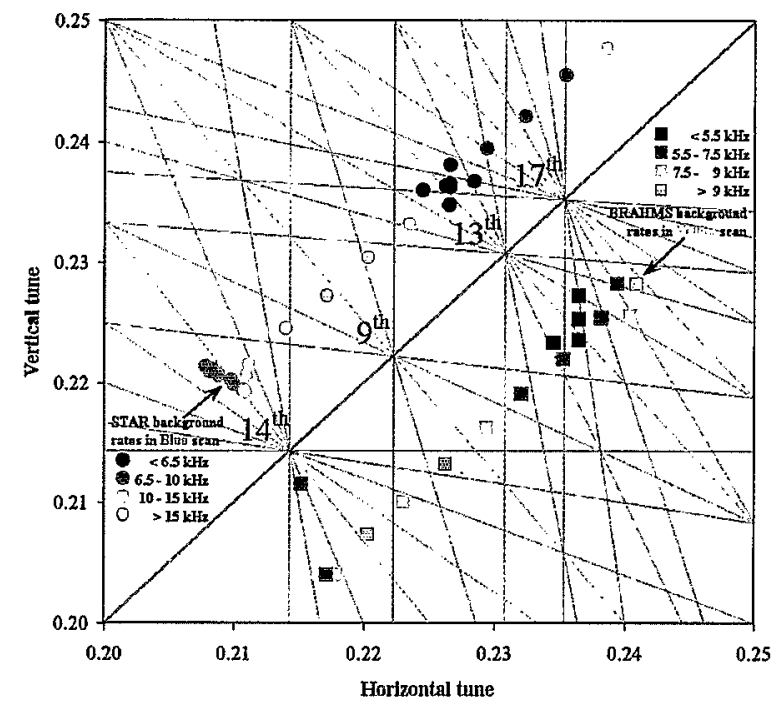

Figure 4: Experimental background rates as a function of fractional tunes. For these measurements deuterons in the Blue ring collide with gold ions in the Yellow ring. The total beam-beam tune spread due to the beam-beam interaction is about $\Delta Q_{\text {tot }}=0.005$ in both measurements.

resonances are of order 9, 13,14 and 17 (Fig. 4). If the nonlinear dynamics are dominated by the beam-beam interaction and the crossing angles are all zero, no odd-order resonances are driven.

Experimental background rates were observed as a function of the tunes with deuteron-gold collisions (Fig. 4). The tunes were moved parallel to the $Q_{x}=Q_{y}$ line, scanning the area considered for operation. With both beams, high background rates were found near $9^{\text {th }}$ order resonances, and low background rates near $13^{\text {th }}$ order resonances. The working points with low background rates are used in operation. High background rates near $9^{\text {th }}$ order resonances are another sign of residual crossing angles (cf. Fig. 2). Increased background rates were also found with a transverse offset [4].

\section{STRONG-STRONG OBSERVATIONS}

RHIC sees strong-strong beam-beam effects. In addition to the tune ( $\sigma$-mode) a new transverse oscillation mode ( $\pi$ mode) occurs. For a single collision per turn the $\pi$-mode is at a tune $Y \xi$ below the $\sigma$-mode, where $Y \approx 1.2$ for round beams [5]. If the beam-beam interaction is the dominant nonlinear effect, the $\pi$-mode can be outside the continuous spectrum and thus be undamped [6].

Coherent beam-beam modes were observed in an experiment with proton beams, with a beam-beam parameter $\xi=0.003$ and a single collision per turn (Fig. 5). The measured difference between the $\sigma$ - and $\pi$-modes is consistent with a Yokoya factor of $Y \approx 1.2$. The locations of the $\pi$-modes were reproduced in a strong-strong simulation [8]. $\pi$-modes were also observed in routine operation with a beam-beam parameter $\xi=0.0015$, four collisions per turn and linear coupling (Fig. 6). The $\pi$-modes could be suppressed by small changes in one of the tunes. 


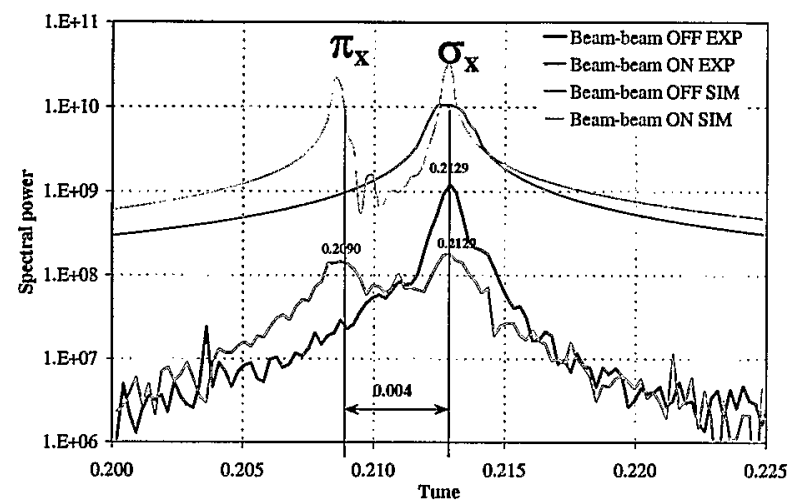

Figure 5: Coherent dipole modes in an experiment with a single proton bunch per beam, and in a corresponding simulation [8]. $\xi=0.003$, spectra from 4096 turns.

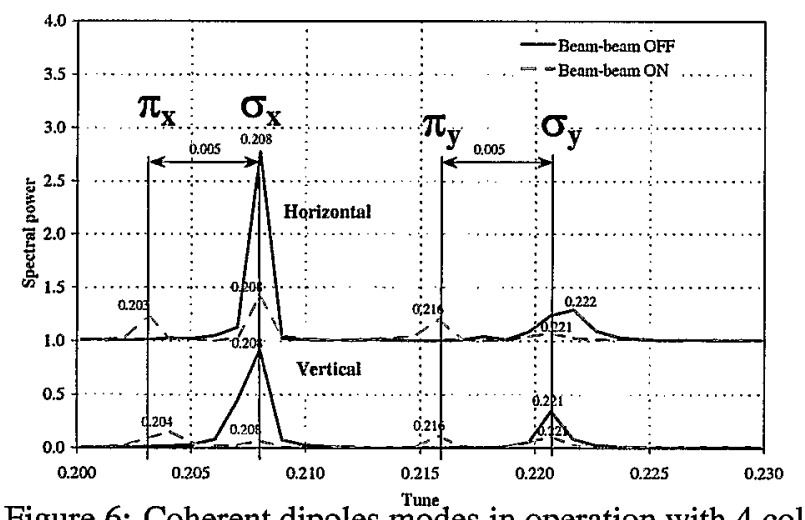

Figure 6: Coherent dipoles modes in operation with 4 collisions per turn. $\xi=0.0015 / \mathrm{IP}$, spectra from 1024 turns.

\section{UNEQUAL RF FREQUENCIES}

When the two RHIC beams have different radio frequencies $f_{r f}$ the beam crossing points move longitudinally with the speed

$$
v_{C P}=\frac{c}{2} \frac{\Delta f_{r f}}{f_{r f}},
$$

where $c$ is the particle speed. Values of $\Delta f_{r f}=5 \mathrm{~Hz}$ and $v_{C P}=27 \mathrm{~m} / \mathrm{s}$ are typical for gold beams in both rings. When deuteron and gold beams were injected with the same rigidity, $\Delta f_{r f}=44 \mathrm{kHz}$ is needed and $v_{C P}=$ $3 \mathrm{~m} /$ turn. Beams experience the beam-beam interaction only when the crossing point is between the DX magnets (Fig. 1). With slowly moving crossing points (gold-gold case) the beam-beam interaction is modulated, with fast moving crossing points (deuteron-gold case) beams experience pseudo random interactions in time.

Slowly moving crossing points and head-on collisions lead to tune modulation and to unacceptable beam lifetime [9]. The tune modulation depth is determined by the beam-beam parameter, the modulation waveform by the crossing angle, and the modulation frequency by the fill pattern and the difference in the rf frequencies $\Delta f_{r f}$. Fig. 7 shows a case typical for gold-gold operation. To avoid this unwanted effect, beams are transversely separated during ramps. For proton beams, which do not pass through transition, the frequencies were locked on the ramp [10].

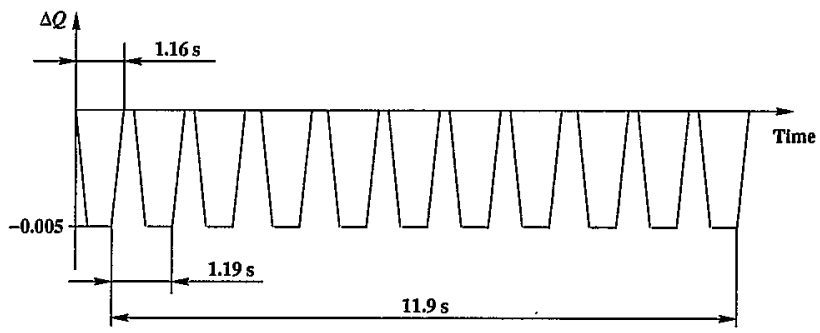

Figure 7: Tune modulation of particles in the center of a bunch due to moving collsion points, for $\Delta f_{r f}=5 \mathrm{~Hz}$, no crossing angle, 60 bunches and a total beam-beam tune shift of $\Delta Q_{\text {tot }}=-0.005$.

The fast moving crossing points with deuteron and gold beams, injected at the same rigidity, lead to unacceptable beam lifetime even with large beam separation. Both beams experienced pseudo-random dipole kicks that lead to emittance growth. Beams had to be injected at the same relativistic $\gamma$, and therefore the same if frequency, to avoid this effect.

\section{SUMMARY}

The beam-beam interaction has a significant impact on lifetime and emittance of the RHIC beams. In addition to beam-beam effects observed in other hadron colliders, coherent beam-beam modes were seen for the first time. With independent $\mathrm{rf}$ systems for both rings, differences in the rf frequencies can lead to tune modulation and emittance growth.

\section{REFERENCES}

[1] T. Roser, "RHIC status and plans", these proceedings.

[2] P. Cameron et al., "RHIC third generation PLL tune system", these proceedings.

[3] S. Saritepe, G. Goderre, and S. Peggs, "Observations of the beam-beam interaction in hadron colliders", in "Frontiers of particle beams: intensity limitations", Springer-Verlag, Lecture Notes in Physics (1991).

[4] A. Drees, $\mathrm{Z}$. Xu, H. Huang, "Resutlts from vernier scans at RHIC during runs 2000-2003", these proceedings.

[5] K. Yokoya and $\mathrm{H}$. Koiso, "Tune shift of coherent beambeam oscillations", Part. Accel. Vol. 27, pp. 181-186 (1990).

[6] Y. Alexahin, "On the Landau Damping and Decoherence of Transverse Dipole Oscillations in Colliding Beams", Part. Accel.,V59, p. 43; CERN-SL-96-064 (AP) (1996).

[7] W. Fischer, L. Ahrens, M. Bai, M. Blaskiewicz, P. Cameron, R. Michnoff, F. Pilat, V. Ptitsyn, T. Sen, S. Tepikian, D. Trbojevic, M. Vogt, and J. van Zeijts, "Observation of coherent beam-beam modes in RHIC", BNL C-A/AP/75 (2002).

[8] M. Vogt, J.A. Ellison, W. Fischer, and T. Sen, "Simulations of coherent beam-beam modes at RHIC", proceedings of the 2002 European Particle Accelerator Conference, Paris (2002).

[9] W. Fischer, P. Cameron, S. Peggs, and T. Satogata, "Tune modulation from beam-beam interaction and unequal radio frequencies in RHIC", BNL C-A/AP/72 (2002).

[10] M. Brennan et al.,"Operation of the RHIC rf system", these proceedings. 